\title{
Quantum Mechanical Effects on Dynamical Behavior of Simple Liquids
}

\author{
Taejun Kim and Hyojoon Kim*
}

\author{
Department of Chemistry, Dong-A University, Busan 604-714, Korea. *E-mail: hkim@donga.ac.kr \\ Received March 24, 2011, Accepted May 18, 2011
}

\begin{abstract}
We evaluate quantum-mechanical velocity autocorrelation functions from classical molecular dynamics simulations using quantum correction approaches. We apply recently developed approaches to supercritical argon and liquid neon. The results show that the methods provide a solution more efficient than previous methods to investigate quantum-mechanical dynamic behavior in condensed phases. Our numerical results are found to be in excellent agreement with the previous quantum-mechanical results.
\end{abstract}

Key Words : Quantum correction approach, Correlation function, Diffusion constant, Quantum dynamics, Quantum-classical calculation

\section{Introduction}

Even with the current computational power, it is not easy to investigate the quantum-mechanical dynamics in condensed phase systems. Therefore, finding more efficient and reliable methods to evaluate quantum-mechanical effects on dynamical behavior in condensed phases has been at the forefront of theoretical chemistry in decades. One of most efficient solutions to calculate quantum-mechanical effects for systems with a large number of degrees of freedom is the quantum correction approach. ${ }^{1-9}$ If we know the analytical relation between the quantum time correlation functions (CF) and their classical counterpart, we can utilize the relation to predict the quantum $\mathrm{CF}$ from the classical simulation data.

Recently, a rigorous quantum correction approach was reported. ${ }^{6-9}$ This approach was based on the rigorous relation between the classical and the quantum CFs unlike other previous quantum correction approaches. It has been successfully applied to the vibration energy relaxation problem, ${ }^{6}$ anharmonic oscillators, ${ }^{7}$ and nonadiabatic transition rates. ${ }^{8}$ The relation between the classical and the Kubotransformed CFs was also found. ${ }^{9}$ In this work, we provide methods to evaluate the quantum velocity time CF from the classical molecular dynamics and apply the methods to simple systems of supercritical argon and liquid neon, where we can measure numerical deviations from the reported quantum results.

\section{Theory}

A formal definition of a general $\mathrm{CF}$ is given by

$$
G(t)=\langle f(A) g(B)\rangle,
$$

where $A$ and $B$ are arbitrary operators and $f$ and $g$ are functions of these operators. According to whether traces are averaged over the quantum or the classical states, the $\mathrm{CF}$ is labeled as $G_{q m}(t)$ or $G_{c l}(t)$, respectively. The relation between $G_{q m}(t)$ and $G_{c l}(t)$ based on the harmonic approximation $\langle\exp (A)\rangle \approx \exp \left[\left\langle A^{2}\right\rangle / 2\right]$ was found as follows: ${ }^{8}$

$$
G_{q m}(t)=\exp \left[\frac{Q\left(\left\langle A^{2}\right\rangle_{c l}\right) \partial}{\partial\left\langle A^{2}\right\rangle_{c l}}+\frac{Q\left(\left\langle B^{2}\right\rangle_{c l}\right) \partial}{\partial\left\langle B^{2}\right\rangle_{c l}}+\frac{Q\left(\langle A B\rangle_{c l}\right) \partial}{\partial\langle A B\rangle_{c l}}\right] G_{c l}(t)
$$

where $Q\left(\langle X\rangle_{c l}\right)=\langle X\rangle_{q m}-\langle X\rangle_{c l}$ is calculable from the corresponding classical CF. ${ }^{6,8}$ Equation (2) is exact when $A$ and $B$ are the position or momentum operators for a harmonic system. ${ }^{8}$ Note that all terms on the right-hand side of Eq. (2) can be evaluated classically.

When we expand Eq. (2) in terms of $\hbar$, we have the following practical expression:

$$
\begin{aligned}
& G_{q m}(t)=G_{c l}(t)+\frac{i \beta \hbar}{2} G_{c l}^{\prime}(t)+\frac{(i \beta \hbar)^{2}}{8} G_{c l}^{\prime \prime}(t) \\
& -\frac{(i \beta \hbar)^{2}}{24} \frac{C_{c l}^{\prime \prime}(t) G_{c l}^{\prime}(t)}{C_{c l}^{\prime}(t)}+\frac{(i \beta \hbar)^{2} C_{c l}^{\prime \prime}(0)}{12} \frac{\partial G_{c l}(t)}{\partial C_{c l}(0)}+O\left(\hbar^{3}\right),
\end{aligned}
$$

where the inverse temperature $\beta=\left(k_{B} T\right)^{-1}$ with the Boltzmann constant $k_{B}$, and $C_{c l}(t)=\langle A B\rangle_{c l}$. Other known approximations are as follows: ${ }^{1-5}$

$$
\begin{aligned}
& \tilde{G}_{\text {standard }}(\omega)=\frac{2}{1+\exp (-\beta \hbar \omega)} \tilde{G}_{c l}(\omega), \\
& \tilde{G}_{\text {harmonic }}(\omega)=\frac{\beta \hbar \omega}{1-\exp (-\beta \hbar \omega)} \tilde{G}_{c l}(\omega), \\
& \tilde{G}_{\text {Schofield }}(\omega)=\exp (\beta \hbar \omega / 2) \tilde{G}_{c l}(\omega), \\
& G_{\text {Egelstaff }}(t)=G_{c l}(\sqrt{t(t+i \beta \hbar)}), \\
& \tilde{G}_{\text {harmonic/Schofield }}(\omega)=\exp (\beta \hbar \omega / 4) \sqrt{\frac{\beta \hbar \omega}{1-\exp (-\beta \hbar \omega)}} \tilde{G}_{c l}(\omega)
\end{aligned}
$$

These approximations except Eq. (7) are given in the Fourier transformed $\tilde{G}(\omega)=\int_{-\infty}^{\infty} d t e^{i \omega t} G(t)$ space.

The accuracy of Eq. (3) is found to be consistently superior to that of other approximations mainly because Eq. (3) provides different expressions for linear and nonlinear CFs unlike other approximations. For the velocity autocorrelation function $G(t)=\langle v(t) v(0)\rangle$, Eq. (3) becomes 


$$
\begin{aligned}
G_{q m}(t)= & G_{c l}(t)+\frac{i \beta \hbar}{2} \frac{d G_{c l}(t)}{d t}+\frac{(i \beta \hbar)^{2}}{12} \frac{d^{2} G_{c l}(t)}{d t^{2}} \\
& -\frac{(i \beta \hbar)^{4}}{720} \frac{d^{4} G_{c l}(t)}{d t^{4}}+O\left(\hbar^{6}\right) .
\end{aligned}
$$

We have utilized the fact that $\partial G_{c l}(t) / \partial C_{c l}(0)=0$ when $G(t)=C(t) .{ }^{6}$ It should be noted that if we sum all the terms in Eq. (9), it reduces to the harmonic approximation Eq. (5) since $G(t)$ is a linear CF. For a linear CF in a harmonic system, Eq. (5) is exact. However, for an anharmonic system, the truncation can actually improve the accuracy. ${ }^{7}$ Furthermore, Eq. (9) is much easier to evaluate than Eq. (5) since it does not involve the numerical Fourier and inverse-Fourier transform, which is often a numerically problematic step.

Note that the only imaginary term of $G_{q m}(t)$ in Eq. (9) is the first order term in $\hbar$ and all other terms are real. Namely,

$$
\operatorname{Im}\left[G_{q m}(t)\right]=\frac{\beta \hbar}{2} \frac{d G_{c l}(t)}{d t}
$$

The quantum CFs can be calculated by truncating Eq. (9) at the order $\hbar^{2}$ or $\hbar^{4}$, respectively, as

$$
\begin{aligned}
& \operatorname{Re}\left[G_{q m}^{2 n d}(t)\right]=G_{c l}(t)-\frac{(\beta \hbar)^{2}}{12} \frac{d^{2} G_{c l}(t)}{d t^{2}}, \\
& \operatorname{Re}\left[G_{q m}^{4 t h}(t)\right]=G_{c l}(t)-\frac{(\beta \hbar)^{2}}{12} \frac{d^{2} G_{c l}(t)}{d t^{2}}-\frac{(\beta \hbar)^{4}}{720} \frac{d^{4} G_{c l}(t)}{d t^{4}} .
\end{aligned}
$$

Before proceeding to the next section, one more comment needs to be made. The Kubo-transformed CF defined as ${ }^{10}$

$$
G_{K}(t)=\langle f(A) ; g(B)\rangle_{q m}=\frac{1}{\beta} \int_{0}^{\beta} d \lambda\left\langle e^{\lambda H} f(A) e^{-\lambda H} g(B)\right\rangle_{q m},
$$

where $H$ is the system Hamiltonian operator, is real. For a linear CF in a harmonic system, $G_{K}(t)=G_{c l}(t) .{ }^{9}$

\section{Results and Discussions}

We perform classical molecular dynamics simulations for Lennard-Jones argon and neon. The simulation parameters are chosen to compare our results with previously reported data. ${ }^{11,12}$ For argon, the Lennard-Jones parameters are set as follows: $\sigma=0.34 \mathrm{~nm} \varepsilon / k_{B}=122.3 \mathrm{~K}$, and $m=6.63 \times 10^{-26}$ $\mathrm{kg}$. We set the density and the temperature as $\rho \sigma^{3}=0.95$ and $k_{B} T / \varepsilon=1.50$, respectively. For these parameters, argon is in the supercritical region. The number of argon atoms realized in simulations is $N=500$. For neon liquids, the parameters are chosen as $N=108, \sigma=0.2749 \mathrm{~nm}, \varepsilon / k_{B}=35.6 \mathrm{~K}, m=$ $3.35 \times 10^{-26} \mathrm{~kg}, \rho \sigma^{3}=0.78$, and $k_{B} T / \varepsilon=0.84$. The classical velocity autocorrelation function $G_{c l}(t)=\langle v(t) v(0)\rangle_{c l}$ is calculated using the DL_POLY program ${ }^{13}$ and the quantum CFs are calculated from the methods in the previous section. With a time step of $1 \mathrm{fs}$, the systems are propagated classically for $100 \mathrm{ps}$ after initial equilibrations.

In Figure 1, the classical and other quantum CFs are compared with the quantum result. ${ }^{11,12}$ One can see that the quantum effects of argon at the temperature are small and there are little differences between classical and quantum

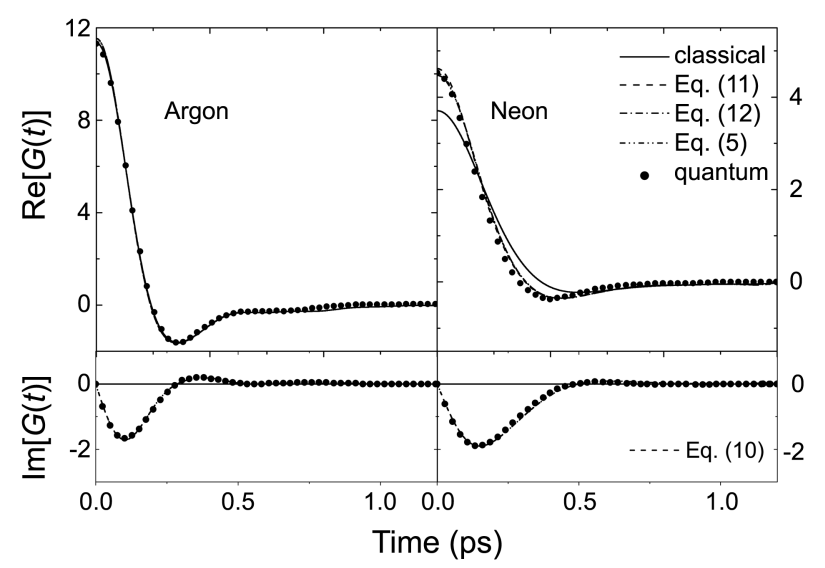

Figure 1. Real and imaginary parts of the velocity autocorrelation functions for argon (left) and neon (right). The classical results are obtained from the classical molecular dynamics simulations using the DL_POLY program, ${ }^{13}$ and the quantum results are obtained from the forward-backward semi-classical dynamics. ${ }^{11,12}$ The units of $G(t)$ are $\AA / \mathrm{ps}^{2}$.

results. Therefore, the second and the fourth order correction methods as well as the harmonic approximation provide correct results for argon. On the other hand, non-negligible differences are found for neon mainly because of the lower temperature. Since the quantum-mechanical velocity distribution is wider than that of the classical mechanics, $G_{q m}(0)$ is larger than $G_{c l}(0)$. The quantum $\mathrm{CF}$ relaxes faster than the classical one. The figure shows that the second order term in $\hbar,(\beta \hbar)^{2} G_{c l}^{\prime \prime}(t) / 12$ gives the most contribution to the quantum effects of neon and the magnitude of higher order terms is relatively small. One may utilize this second order term as a practical check for the magnitude of quantum effects of the interested system. We evaluate Eq. (5) by performing the numerical Fourier transform $G_{c l}(t)$, multiplying the factor, and then performing the inverse Fourier transform. Equation (11) is much easier to obtain the time domain results with little loss of accuracy. Note that the classical CF has no imaginary value. As mentioned in the earlier section, the only imaginary part in the quantum $\mathrm{CF}$ is the first order term in $\hbar$. This term gives accurate descriptions for both argon and neon.

We have also compared the $\mathrm{CF}$ in the Fourier-transformed domain in Figure 2. Even though the supercritical argon is nearly classical, the difference between the classical and quantum results in the frequency space is non-negligible due to the imaginary term. Note that $\tilde{G}_{c l}(\omega)$ is an even function. Again, the contribution of higher order terms than $\hbar^{2}$ is small and Eq. (11) is in good agreement with the quantum results for both argon and neon.

Since the diffusion constant is related to the $\mathrm{CF}$ as $D=(1 /$ 3) $\int d t G(t)$, it is interesting to investigate quantum effects on $D$. Both of the classical and the quantum diffusion constants of argon are found to be $D=2.571 \times 10^{-5} \mathrm{~cm}^{2} \cdot \mathrm{sec}^{-1}$. For neon, $D_{c l}=1.945 \times 10^{-5} \mathrm{~cm}^{2} \cdot \mathrm{sec}^{-1}, D_{2 n d}=1.937 \times 10^{-5} \mathrm{~cm}^{2} \cdot \mathrm{sec}^{-1}$, $D_{4 t h}=1.940 \times 10^{-5} \mathrm{~cm}^{2} \cdot \mathrm{sec}^{-1}$, and $D_{\text {harmonic }}=1.939 \times 10^{-5}$ $\mathrm{cm}^{2} \cdot \mathrm{sec}^{-1}$. The ratio $D_{2 n d} / D_{c l}$ is about 0.996 . Even though the dynamics of neon shows the significant quantum effects, the 


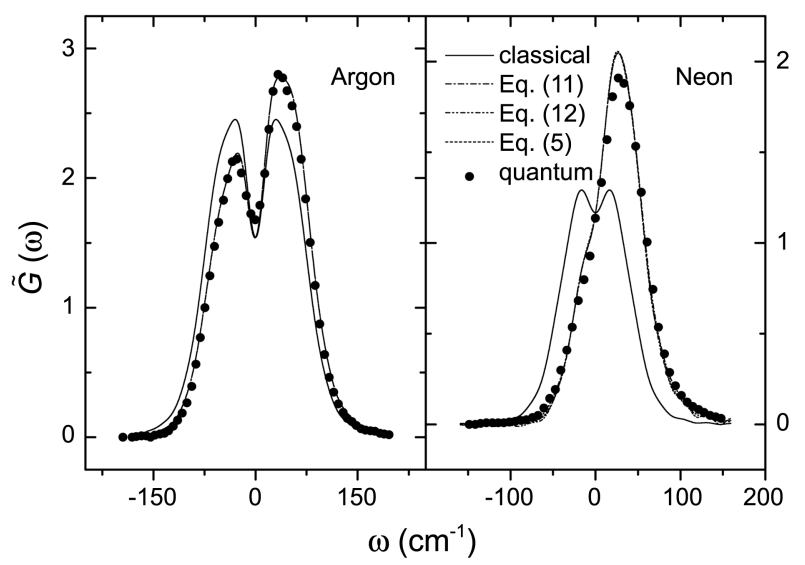

Figure 2. Fourier transform of the velocity autocorrelation functions for argon (left) and neon (right). The units of $\tilde{G}(\omega)$ are $\AA / p s$.

diffusion constant is not affected since two quantum effects of the initial large velocity distribution and the fast relaxation are complementary. This is consistent with the fact that $G_{K}(t)=G_{c l}(t)$.

One of main advantages of computer simulations is that we can investigate a specific effect straightforwardly by changing only one parameter. We increase only the density of neon atoms from $\rho \sigma^{3}=0.78$ to 0.87 without changing other parameters. In Figure 3, one can see that the higher density causes the larger quantum effects. The calculated diffusion constants are reduced as follows: $D_{c l}=1.212 \times$ $10^{-5} \mathrm{~cm}^{2} \cdot \mathrm{sec}^{-1}, D_{2 n d}=1.207 \times 10^{-5} \mathrm{~cm}^{2} \cdot \mathrm{sec}^{-1}, D_{4 t h}=1.208 \times$ $10^{-5} \mathrm{~cm}^{2} \cdot \mathrm{sec}^{-1}$, and $D_{\text {harmonic }}=1.208 \times 10^{-5} \mathrm{~cm}^{2} \cdot \mathrm{sec}^{-1}$. However, the ratio is not changed, namely, $D_{2 n d} / D_{c l}=0.996$. As Figure 4 shows, the trends in the frequency space are also similar to those in Figure 2. The second order solution is

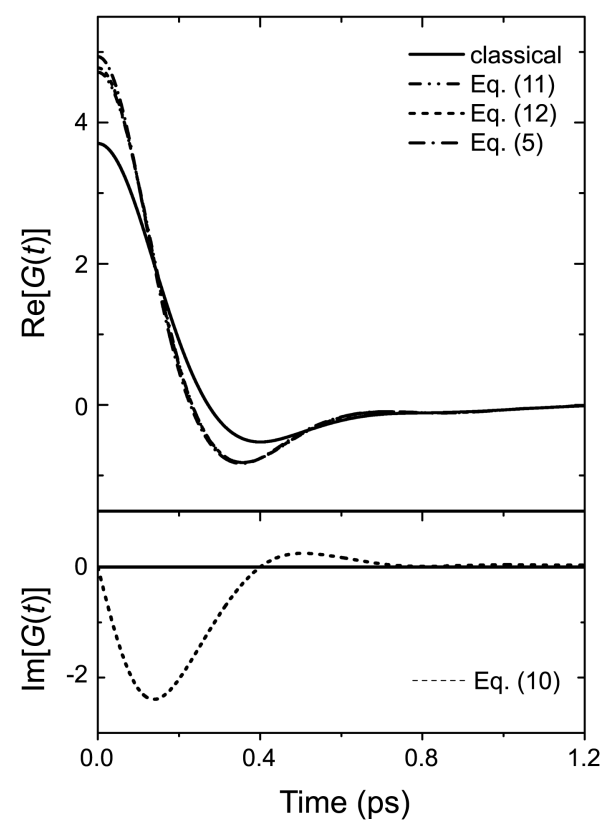

Figure 3. Real and imaginary parts of the velocity autocorrelation function for neon at the increased density of 0.87 . The units of $G(t)$ are $\AA / \mathrm{ps}^{2}$.

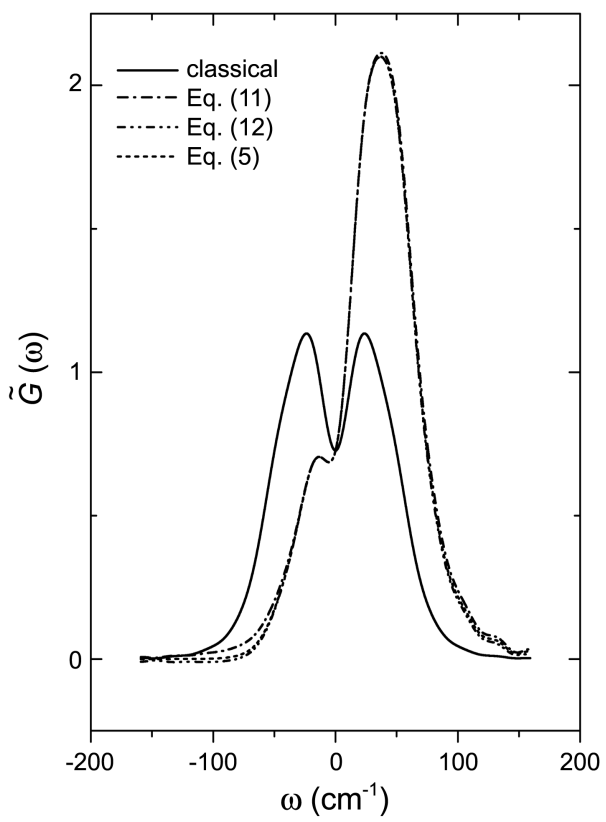

Figure 4. Fourier transform of the velocity autocorrelation function for neon at the increased density of 0.87 . The units of $\tilde{G}(\omega)$ are $\AA$ / ps.

found to be still accurate in the system with larger quantum effects.

We perform simulations of neon at the higher densities and at the lower temperatures. We find that $D_{2 n d} / D_{c l}$ is not affected by the increased quantum behavior. (Actually, the ratio tends to decrease very slightly.) When the density is further increased or the temperature is further decreased, the phase transition seems to occur and the ratio decreases significantly. We find that $D_{2 n d} / D_{c l}=0.98$ at $k_{B} T / \varepsilon=0.35$, and $D_{2 n d} / D_{c l}=0.96$ at $\rho \sigma^{3}=1.00$. This is an observation seemingly opposite to those in the previous reports that the quantum diffusion constant was bigger than the classical one. ${ }^{14,15}$ This may result from the phase transition or the limitation of the quantum correction approach. The detailed analysis will be reported elsewhere.

\section{Concluding Remarks}

We have investigated quantum-mechanical effects on dynamical behavior in supercritical argon and liquid neon. From the classical molecular dynamics simulations, we evaluate quantum effects on the velocity autocorrelation functions using quantum correction approaches. For a variety of systems, our new approach, based on the rigorous relation between classical and quantum correlation functions, shows high efficiency with reliable accuracy. For the linear correlation function studied in the present work, the approach can reduce to the harmonic approximation. Truncated series solutions, which do not necessitate numerical Fourier and inverse-Fourier transforms, are found to reproduce the quantum results more efficiently. Especially, the second order solution is much more efficient with little loss of accuracy than the harmonic approximation. 
As in other previous reports, the supercritical argon is nearly classical and liquid neon shows significant quantummechanical behavior. The quantum neon shows the larger initial velocity distribution and the faster velocity relaxation than the classical neon. The dynamical quantum behavior is well described by the current quantum correction approach. However, the quantum effects on the diffusion constant are not noticeable since the two quantum effects are complementary.

Acknowledgments. This work was supported by research funds from Dong-A University.

\section{References}

1. Schofield, P. Phys. Rev. Lett. 1960, 4, 239.
2. Egelstaff, P. A. Adv. Phys. 1962, 11, 203.

3. Berne, B. J.; Harp, G. D. Adv. Chem. Phys. 1970, 17, 63

4. Berens, P. H.; White, S. R.; Wilson, K. R. J. Chem. Phys. 1981, 75,515 .

5. Egorov, S. A.; Everitt, K. F.; Skinner, J. L. J. Phys. Chem. A 1999, 103, 9494 .

6. Kim, H.; Rossky, P. J. J. Phys. Chem. B 2002, 106, 8240.

7. Kim, H.; Rossky, P. J. J. Chem. Phys. 2006, 125, 074107.

8. Kim, H.; Rossky, P. J. J. Chem. Phys. 2006, 125, 066101.

9. Kim, H. Chem. Phys. Lett. 2007, 436, 111.

10. Zwanzig, R. Annu. Rev. Phys. Chem. 1965, 16, 67.

11. Wright, N. J.; Makri, N. J. Chem. Phys 2003, 119, 1634.

12. Lawrence, C. P.; Nakayama, A.; Makri, N.; Skinner, J. L. J. Chem. Phys. 2004, 120, 6621.

13. Smith, W.; Forester, T. R. J. Mol. Graphics 1996, 14, 136.

14. Poulsen, J. A.; Nyman, G.; Rossky, P. J. J. Phys. Chem. B 2004, $108,19799$.

15. Miller III, T. F.; Manolopoulos, D. E. J. Chem. Phys. 2005, 122, 154504 . 